\title{
Some algorithms for equilibrium problems on Hadamard manifolds
}

\author{
Muhammad Aslam Noor ${ }^{*}$ and Khalida Inayat Noor
}

${ }^{\text {*Correspondence: }}$

noormaslam@hotmail.com

Mathematics Department,

COMSATS Institute of Information

Technology, Park Road, Islamabad,

Pakistan

\begin{abstract}
In this paper, we suggest and analyze an iterative method for solving the equilibrium problems on Hadamard manifolds using the auxiliary principle technique. We also consider the convergence analysis of the proposed method under suitable conditions. Some special cases are considered. Results and ideas of this paper may stimulate further research in this fascinating and interesting field.
\end{abstract}

MSC: 49J40; 90C33; 26D10; 39B62

Keywords: variational inequalities; Hadamard manifold; implicit methods; convergence

\section{Introduction}

Equilibrium problems theory provides us with a unified, natural, novel and general framework to study a wide class of problems, which arise in finance, economics, network analysis, transportation and optimization. This theory has applications across all disciplines of pure and applied sciences. Equilibrium problems include variational inequalities and related problems as special cases; see [1-31]. Recently, much attention has been given to study the variational inequalities, equilibrium and related optimization problems on the Riemannian manifold and Hadamard manifold. This framework is useful for the development of various fields. Several ideas and techniques from the Euclidean space have been extended and generalized to this nonlinear framework. Hadamard manifolds are examples of hyperbolic spaces and geodesics; see $[1,3-5,19,20,26-28]$ and the references therein. Nemeth [8], Tang et al. [28], Noor et al. [19, 20] and Colao et al. [3] have considered the variational inequalities and equilibrium problems on Hadamard manifolds. They have studied the existence of solutions of equilibrium problems under some suitable conditions. To the best of our knowledge, no one has considered the auxiliary principle technique for solving the equilibrium problems on Hadamard manifolds. In this paper, we use the auxiliary principle technique to suggest and analyze an implicit method for solving the equilibrium problems on a Hadamard manifold. As special cases, our result includes the recent results of Tang et al. [28] for variational inequalities on a Hadamard manifold. This shows that the results obtained in this paper continue to hold for variational inequalities on a Hadamard manifold, which are due to Noor and Noor [20], Tang et al. [28], and Nemeth [8]. We hope that the technique and idea of this paper may stimulate further research in this area.

(C) 2012 Noor and Noor; licensee Springer. This is an Open Access article distributed under the terms of the Creative Commons Attribution License (http://creativecommons.org/licenses/by/2.0), which permits unrestricted use, distribution, and reproduction in any medium, provided the original work is properly cited. 


\section{Preliminaries}

We now recall some fundamental and basic concepts needed for reading of this paper. These results and concepts can be found in the books on Riemannian geometry $[1,3,4,26,29]$.

Let $M$ be a simply connected $m$-dimensional manifold. Given $x \in M$, the tangent space of $M$ at $x$ is denoted by $T_{x} M$ and the tangent bundle of $M$ by $T M=\bigcup_{x \in M} T_{x} M$, which is naturally a manifold. A vector field $A$ on $M$ is a mapping of $M$ into TM which associates to each point $x \in M$, a vector $A(x) \in T_{x} M$. We always assume that $M$ can be endowed with a Riemannian metric to become a Riemannian manifold. We denote by $\langle\cdot, \cdot\rangle$ the scalar product on $T_{x} M$ with the associated norm $\|\cdot\|_{x}$, where the subscript $x$ will be omitted. Given a piecewise smooth curve $\gamma:[a, b] \longrightarrow M$ joining $x$ to $y$ (that is, $\gamma(a)=x$ and $\gamma(b)=y$ ) by using the metric, we can define the length of $\gamma$ as $L(\gamma)=\int_{a}^{b}\left\|\gamma^{\prime}(t)\right\| d t$. Then for any $x, y \in M$, the Riemannian distance $d(x, y)$, which includes the original topology on $M$, is defined by minimizing this length over the set of all such curves joining $x$ to $y$.

Let $\Delta$ be the Levi-Civita connection with $(M,\langle\cdot, \cdot\rangle)$. Let $\gamma$ be a smooth curve in $M$. A vector field $A$ is said to be parallel along $\gamma$ if $\Delta_{\gamma^{\prime}} A=0$. If $\gamma^{\prime}$ itself is parallel along $\gamma$, we say that $\gamma$ is a geodesic and in this case $\left\|\gamma^{\prime}\right\|$ is a constant. When $\left\|\gamma^{\prime}\right\|=1, \gamma$ is said to be normalized. A geodesic joining $x$ to $y$ in $M$ is said to be minimal if its length equals $d(x, y)$.

A Riemannian manifold is complete if for any $x \in M$, all geodesics emanating from $x$ are defined for all $t \in R$. By the Hopf-Rinow theorem, we know that if $M$ is complete, then any pair of points in $M$ can be joined by a minimal geodesic. Moreover, $(M, d)$ is a complete metric space, and bounded closed subsets are compact.

Let $M$ be complete. Then the exponential map $\exp _{x}: T_{x} M \longrightarrow M$ at $x$ is defined by $\exp _{x} v=\gamma_{v}(1, x)$ for each $v \in T_{x} M$, where $\gamma(\cdot)=\gamma_{v}(\cdot, x)$ is the geodesic starting at $x$ with velocity $v\left(\right.$ i.e., $\gamma(0)=x$ and $\left.\gamma^{\prime}(0)=v\right)$. Then $\exp _{x} t v=\gamma_{v}(t, x)$ for each real number $t$.

A complete simply connected Riemannian manifold of nonpositive sectional curvature is called a Hadamard manifold. Throughout the remainder of this paper, we always assume that $M$ is an $m$-dimensional Hadamard manifold.

We also recall the following well-known results, which are essential for our work.

Lemma 2.1 ([26]) Let $x \in M$. Then $\exp _{x}: T_{x} M \rightarrow M$ is a diffeomorphism, and for any two points $x, y \in M$, there exists a unique normalized geodesic joining $x$ to $y, \gamma_{x, y}$, which is minimal.

So, from now on, when referring to the geodesic joining two points, we mean the unique minimal normalized one. Lemma 2.1 says that $M$ is diffeomorphic to the Euclidean space $R^{m}$. Thus, $M$ has the same topology and differential structure as $R^{m}$. It is also known that Hadamard manifolds and Euclidean spaces have similar geometrical properties. Recall that a geodesic triangle $\Delta\left(x_{1}, x_{2}, x_{3}\right)$ of a Riemannian manifold is a set consisting of three points $x_{1}, x_{2}, x_{3}$ and three minimal geodesics joining these points.

Lemma 2.2 (Comparison theorem for triangles $[3,4,26])$ Let $\triangle\left(x_{1}, x_{2}, x_{3}\right)$ be a geodesic triangle. Denote, for each $i=1,2,3(\bmod 3)$, by $\gamma_{i}:\left[0, l_{i}\right] \longrightarrow M$ the geodesic joining $x_{i}$ to $x_{i+1}$, and $\alpha_{i} ;=L\left(\gamma_{i}^{\prime}(0),-\gamma_{l}^{\prime}(i-1)(l i-1)\right)$, the angle between the vectors $\gamma_{i}^{\prime}(0)$ and $-\gamma_{i-1}^{\prime}\left(l_{i-1}\right)$, 
and $l_{i} ;=L\left(\gamma_{i}\right)$. Then

$$
\begin{aligned}
& \alpha_{1}+\alpha_{2}+\alpha_{3} \leq \pi, \\
& l_{l}^{2}+l_{i+1}^{2}-2 L_{i} ; l_{i+1} \cos \alpha_{i+1} \leq l_{i-1}^{2} .
\end{aligned}
$$

In terms of the distance and the exponential map, the inequality (2.2) can be rewritten as

$$
d^{2}\left(x_{i}, x_{i+1}\right)+d^{2}\left(x_{i+1}, x_{i+2}\right)-2\left\langle\exp _{x_{i+1}}^{-1} x_{i}, \exp _{x_{i+1}}^{-1} x_{i+2}\right\rangle \leq d^{2}\left(x_{i-1}, x_{i}\right)
$$

since

$$
\left\langle\exp _{x_{i+1}}^{-1} x_{i}, \exp _{x_{i+1}}^{-1} x_{i+2}\right\rangle=d\left(x_{i}, x_{i+1}\right) d\left(x_{i+1}, x_{i+2}\right) \cos \alpha_{i+1} \text {. }
$$

Lemma 2.3 ([26]) Let $\triangle(x, y, z)$ be a geodesic triangle in a Hadamard manifold $M$. Then there exist $x^{\prime}, y^{\prime}, z^{\prime} \in R^{2}$ such that

$$
d(x, y)=\left\|x^{\prime}-y^{\prime}\right\|, \quad d(y, z)=\left\|y^{\prime}-z^{\prime}\right\|, \quad d(z, x)=\left\|z^{\prime}-x^{\prime}\right\| .
$$

The triangle $\triangle\left(x^{\prime}, y^{\prime}, z^{\prime}\right)$ is called the comparison triangle of the geodesic triangle $\triangle(x, y, z)$, which is unique up to isometry of $M$.

From the law of cosines in inequality (2.3), we have the following inequality, which is a general characteristic of the spaces with nonpositive curvature [26]:

$$
\left\langle\exp _{x}^{-1} y, \exp _{x}^{-1} z\right\rangle+\left\langle\exp _{y}^{-1} x, \exp _{y}^{-1} z\right\rangle \geq d^{2}(x, y)
$$

From the properties of the exponential map, we have the following known result.

Lemma 2.4 ([26]) Let $x_{0} \in M$ and $\left\{x_{n}\right\} \subset M$ such that $x_{n} \longrightarrow x_{0}$. Then the following assertions hold.

(i) For any $y \in M$,

$$
\exp _{x_{n}}^{-1} y \longrightarrow \exp _{x_{o}}^{-1} y \text { and } \exp _{y}^{-1} x_{n} \longrightarrow \exp _{y}^{-1} x_{o}
$$

(ii) If $\left\{v_{n}\right\}$ is a sequence such that $v_{n} \in T_{x_{n}} M$ and $v_{n} \rightarrow v_{0}$, then $v_{0} \in T_{x_{0}} M$.

(iii) Given the sequences $\left\{u_{n}\right\}$ and $\left\{v_{n}\right\}$ satisfying $u_{n}, v_{n} \in T_{x_{n}} M$, if $u_{n} \longrightarrow u_{0}$ and $v_{n} \longrightarrow v_{0}$, with $u_{0}, v_{0} \in T_{x_{0}} M$, then

$$
\left\langle u_{n}, v_{n}\right\rangle \longrightarrow\left\langle u_{0}, v_{0}\right\rangle
$$

A subset $K \subseteq M$ is said to be convex if for any two points $x, y \in K$, the geodesic joining $x$ and $y$ is contained in $K$, that is, if $\gamma:[a, b] \longrightarrow M$ is a geodesic such that $x=\gamma(a)$ and $y=\gamma(b)$, then $\gamma((1-t) a+t b) \in K, \forall t \in[0,1]$. From now on, $K \subseteq M$ will denote a nonempty, closed and convex set, unless explicitly stated otherwise. 
A real-valued function $f$ defined on $K$ is said to be convex, if for any geodesic $\gamma$ of $M$, the composition function $f \circ \gamma: R \longrightarrow R$ is convex, that is,

$$
(f \circ \gamma)(t a+(1-t) b) \leq t(f \circ \gamma)(a)+(1-t)(f \circ \gamma)(b), \quad \forall a, b \in R, t \in[0,1]
$$

The subdifferential of a function $f: M \longrightarrow R$ is the set-valued mapping $\partial f: M \longrightarrow 2^{T M}$ defined as

$$
\partial f(x)=\left\{u \in T_{x} M:\left\langle u, \exp _{x}^{-1} y\right\rangle \leq f(y)-f(x), \forall y \in M\right\}, \quad \forall x \in M,
$$

and its elements are called subgradients. The subdifferential $\partial f(x)$ at a point $x \in M$ is a closed and convex (possibly empty) set. Let $D(\partial f)$ denote the domain of $\partial f$ defined by

$$
D(\partial f)=\{x \in M: \partial f(x) \neq \emptyset\} .
$$

The existence of subgradients for convex functions is guaranteed by the following proposition, see [29].

Lemma 2.5 ([26, 29]) Let $M$ be a Hadamard manifold and $f: M \rightarrow R$ be convex. Then for any $x \in M$, the subdifferential $\partial f(x)$ of $f$ at $x$ is nonempty. That is, $D(\partial f)=M$.

For a given bifunction $F(\cdot, \cdot): K \times K \longrightarrow R$, we consider the problem of finding $u \in K$ such that

$$
F(u, v) \geq 0, \quad \forall v \in K,
$$

which is called the equilibrium problem on Hadamard manifolds. This problem was considered by Colao et al. [3]. They proved the existence of a solution of the problem (2.5) using the KKM maps. Colao et al. [3] have given an example of the equilibrium problem defined in an Euclidean space whose set $K$ is not a convex set, so it cannot be solved using the technique of Blum and Oettli [2]. However, if one can reformulate the equilibrium problem on a Riemannian manifold, then it can be solved. This shows the importance of considering these problems on Hadamard manifolds. Noor et al. $[19,20]$ have used the auxiliary principle technique to suggest and analyze an implicit method for solving the equilibrium problems on a Hadamard manifold. For the applications, formulation and other aspects of equilibrium problems in the linear setting, see [2, 5, 7-18, 22].

If $F(u, v)=\left\langle T u, \exp _{u}^{-1} v\right\rangle$, where $T: K \rightarrow T M$ is a single-valued vector filed, then problem (2.5) is equivalent to finding $u \in K$ such that

$$
\left\langle T u, \exp _{x}^{-1} v\right\rangle \geq 0, \quad \forall v \in K
$$

which is called the variational inequality on Hadamard manifolds. Nemeth [8], Colao et al. [3], Tang et al. [28] and Noor and Noor [19] studied variational inequalities on a Hadamard manifold from different points of view. In the linear setting, variational inequalities have been studied extensively; see [2, 6, 9-22, 27, 30, 31] and the references therein. 
Definition 2.1 A bifunction $F(\cdot, \cdot)$ is said to be partially relaxed strongly monotone if and only if there exists a constant $\alpha>0$ such that

$$
F(u, v)+F(v, z) \leq \alpha d^{2}(z, u), \quad \forall u, v, z \in K .
$$

We note that if $z=u$, then partially relaxed strongly monotonicity reduces to the monotonicity of the bifunction $F(\cdot, \cdot)$.

\section{Main results}

We now use the auxiliary principle technique of Glowinski et al. [6] to suggest and analyze an implicit iterative method for solving the equilibrium problems (2.5).

For a given $u \in K$ satisfying (2.5), consider the problem of finding $w \in K$ such that

$$
\rho F(u, v)+\left\langle\exp _{u}^{-1} w, \exp _{w}^{-1} v\right\rangle \geq 0, \quad \forall v \in K,
$$

which is called the auxiliary equilibrium problem on Hadamard manifolds. We note that if $w=u$, then $w$ is a solution of (2.5). This observation enables us to suggest and analyze the following implicit method for solving the equilibrium problems (2.5). This is the main motivation of this paper.

Algorithm 3.1 For a given $u_{0}$, compute the approximate solution by the iterative scheme

$$
\rho F\left(u_{n}, v\right)+\left\langle\exp _{u_{n}}^{-1} u_{n+1}, \exp _{u_{n+1}}^{-1} v\right\rangle \geq 0, \quad \forall v \in K .
$$

Algorithm 3.1 is called the explicit iterative method for solving the equilibrium problem on the Hadamard manifold.

If $K$ is a convex set in $R^{n}$, then Algorithm 3.1 collapses to

Algorithm 3.2 For a given $u_{0} \in K$, find the approximate solution $u_{n+1}$ by the iterative scheme

$$
\rho F\left(u_{n}, v\right)+\left\langle u_{n+1}-u_{n}, v-u_{n+1}\right\rangle \geq 0, \quad \forall v \in K,
$$

which is known as the explicit method for solving the equilibrium problem. For the convergence analysis of Algorithm 3.2, see [12, 15, 16].

If $F(u, v)=\left\langle T u, \exp _{u}^{-1} v\right\rangle$, where $T$ is a single valued vector filed $T: K \longrightarrow T M$, then Algorithm 3.1 reduces to the following implicit method for solving the variational inequalities.

Algorithm 3.3 For a given $u_{0} \in K$, compute the approximate solution $u_{n+1}$ by the iterative scheme

$$
\left\langle\rho T u_{n}+\left(\exp _{u_{n}}^{-1} u_{n+1}\right), \exp _{u_{n+1}}^{-1} v\right\rangle \geq 0, \quad \forall v \in K
$$

For $M=R^{n}$, Algorithm 3.3 reduces to 
Algorithm 3.4 For a given $u_{0} \in K$, compute the approximate solution $u_{n+1}$ by the iterative scheme

$$
\left\langle\rho T u_{n}+u_{n+1}-u_{n}, v-u_{n+1}\right\rangle \geq 0, \quad \forall v \in K,
$$

which can be written in the following equivalent form.

Algorithm 3.5 For a given $u_{0} \in K$, compute the approximate solution $u_{n+1}$ by the iterative scheme

$$
u_{n+1}=P_{K}\left[u_{n}-\rho T u_{n}\right], \quad n=0,1,2, \ldots
$$

which is known as the projection method. For the convergence analysis and its applications, see $[10,11]$.

In a similar way, one can obtain several iterative methods for solving the variational inequalities on the Hadamard manifold.

We now consider the convergence analysis of Algorithm 3.1, and this is the motivation of our next result.

Theorem 3.1 Let $F(\cdot, \cdot)$ be a partially relaxed strongly monotone bifunction with a constant $\alpha>0$. Let $u_{n}$ be the approximate solution of the equilibrium problem (2.5) obtained from Algorithm 3.1, then

$$
d^{2}\left(u_{n+1}, u\right) \leq d^{2}\left(u_{n}, u\right)-(1-\rho \alpha) d^{2}\left(u_{n+1}, u_{n}\right)
$$

where $u \in K$ is a solution of the equilibrium problem (2.5).

Proof Let $u \in K$ be a solution of the equilibrium problem (2.5). Then

$$
F(u, v) \geq 0, \quad \forall v \in K
$$

Taking $v=u_{n+1}$ in (3.4), we have

$$
F\left(u, u_{n+1}\right) \geq 0
$$

Taking $v=u$ in (3.2), we have

$$
\rho F\left(u_{n}, u\right)+\left\langle\exp _{u_{n}}^{-1} u_{n+1}, \exp _{u_{n+1}}^{-1} u\right\rangle \geq 0
$$

From (3.5) and (3.6), we have

$$
\begin{aligned}
\left\langle\exp _{u_{n+1}}^{-1} u_{n}, \exp _{u_{n+1}}^{-1} u\right\rangle & \leq\left\{F\left(u, u_{n+1}\right)+F\left(u_{n}, u\right)\right\} \\
& \leq \alpha \rho d^{2}\left(u_{n+1}, u_{n}\right)
\end{aligned}
$$


where we have used the fact that the bifunction $F(\cdot, \cdot)$ is partially relaxed strongly monotone with a constant $\alpha>0$. For the geodesic triangle $\Delta\left(u_{n}, u_{n+1}, u\right)$, the inequality (3.7) can be written as

$$
d^{2}\left(u_{n+1}, u\right)+d^{2}\left(u_{n+1}, u_{n}\right)-\left\langle\exp _{u_{n+1}}^{-1} u_{n}, \exp _{u_{n+1}}^{-1} u\right\rangle \leq d^{2}\left(u_{n}, u\right)
$$

Thus, from (3.7) and (3.8), we obtained the inequality (3.3), the required result.

Theorem 3.2 Let $u \in K$ be solution of (2.5), and let $u_{n+1}$ be the approximate solution obtained from Algorithm 3.1. If $\rho<\frac{1}{2 \alpha}$, then $\lim _{n \rightarrow \infty} u_{n+1}=u$.

Proof Let $\hat{u}$ be a solution of (2.5). Then, from (3.3), it follows that the sequence $\left\{u_{n}\right\}$ is bounded and

$$
\sum_{n=0}^{\infty}(1-2 \alpha \rho) d^{2}\left(u_{n+1}, u_{n}\right) \leq d^{2}\left(u_{0}, u\right)
$$

from which, we have

$$
\lim _{n \rightarrow \infty} d\left(u_{n+1}, u_{n}\right)=0 .
$$

Let $\hat{u}$ be a cluster point of $\left\{u_{n}\right\}$. Then there exits a subsequence $\left\{u_{n_{i}}\right\}$ such that $\left\{u_{u_{i}}\right\}$ converges to $\hat{u}$. Replacing $u_{n+1}$ by $u_{n_{i}}$ in (3.2), taking the limit and using (3.9), we have

$$
F(\hat{u}, v) \geq 0, \quad \forall v \in K \text {. }
$$

This shows that $\hat{u} \in K$ solves (2.5) and

$$
d^{2}\left(u_{n+1}, \hat{u}\right) \leq d^{2}\left(u_{n}, \hat{u}\right)
$$

which implies that the sequence $\left\{u_{n}\right\}$ has a unique cluster point and $\lim _{n \rightarrow \infty} u_{n}=\hat{u}$ is a solution of (2.5), the required result.

\section{Conclusion}

The auxiliary principle technique is used to suggest and analyze an explicit method for solving the equilibrium problems on Hadamard manifolds. It is shown that the convergence analysis of this method requires only the partially relaxed strongly monotonicity. Some special cases are discussed. Results proved in this paper may stimulate research in this area. 


\section{Acknowledgements}

The authors would like to thank Dr. S. M. Junaid Zaidi, Rector, COMSATS Institute of Information Technology, Islamabad, Pakistan, for providing excellent research facilities. The authors are grateful to the referees for their constructive suggestions and comments.

Received: 27 April 2012 Accepted: 28 September 2012 Published: 12 October 2012

\section{References}

1. Azagra, D, Ferrera, J, Lopez-Mesas, F: Nonsmooth analysis and Hamiltonian-Jacobi equations on Riemannian manifolds. J. Funct. Anal. 220, 304-361 (2005)

2. Blum, E, Oettli, W: From optimization and variational inequalities to equilibrium problems. Math. Stud. 63, 123-145 (1994)

3. Colao, V, Lopez, G, Marino, G, Martin-Marquez, V: Equilibrium problems in Hadamard manifolds. J. Math. Anal. Appl. $388,61-77(2012)$

4. DoCarmo, MP: Riemannian Geometry. Birkhäuser, Boston (1992)

5. Ferrera, OP, Oliveira, PR: Proximal point algorithms on Riemannian manifolds. Optimization 51(2), 257-270 (2002)

6. Glowinski, R, Lions, JL, Tremolieres, R: Numerical Analysis of Variational Inequalities. North-Holland, Amsterdam (1981)

7. Li, C, Lopez, G, Martin-Marquez, V, Wang, JH: Resolvent of set valued monotone vector fields in Hadamard manifolds. Set-Valued Var. Anal. 19(3), 361-383 (2011)

8. Nemeth, SZ: Variational inequalities on Hadamard manifolds. Nonlinear Anal. 52(5), 1491-1498 (2003)

9. Noor, MA: General variational inequalities. Appl. Math. Lett. 1, 119-121 (1988)

10. Noor, MA: New approximation schemes for general variational inequalities. J. Math. Anal. Appl. 251, 217-229 (2000)

11. Noor, MA: Some developments in general variational inequalities. Appl. Math. Comput. 152, 199-277 (2004)

12. Noor, MA: Fundamentals of mixed quasi variational inequalities. Int. J. Pure Appl. Math. 15, 137-258 (2004)

13. Noor, MA: Auxiliary principle technique for equilibrium problems. J. Optim. Theory Appl. 122, 131-146 (2004)

14. Noor, MA: Fundamentals of equilibrium problems. Math. Inequal. Appl. 9, 529-566 (2006)

15. Noor, MA: Extended general variational inequalities. Appl. Math. Lett. 22, 182-185 (2009)

16. Noor, MA: On an implicit method for nonconvex variational inequalities. J. Optim. Theory Appl. 147, 411-417 (2010)

17. Noor, MA: Auxiliary principle technique for solving general mixed variational inequalities. J. Adv. Math. Stud. 3(2), 89-96 (2010)

18. Noor, MA, Noor, Kl: On equilibrium problems. Appl. Math. E-Notes 4, 125-132 (2004)

19. Noor, MA, Noor, Kl: Proximal point methods for solving mixed variational inequalities on Hadamard manifolds. J. Appl. Math. 2012, Article ID 657278 (2012)

20. Noor, MA, Zainab, S, Yao, Y: Implicit methods for equilibrium problems on Hadamard manifolds. J. Appl. Math. 2012, Article ID 437391 (2012)

21. Noor, MA, Noor, KI, Rassias, TM: Some aspects of variational inequalities. J. Comput. Appl. Math. 47, 285-312 (1993)

22. Noor, MA, Oettli, W: On general nonlinear complementarity problems and quasi equilibria. Matematiche 49, 313-331 (1994)

23. Pitea, A, Postolache, M: Duality theorems for a new class multitime multiobjective variational problems. J. Glob. Optim. 54(1), 47-58 (2012)

24. Pitea, A, Postolache, M: Minimization of vectors of curvilinear functionals on the second order jet: necessary conditions. Optim. Lett. 6(3), 459-470 (2012)

25. Pitea, A, Postolache, M: Minimization of vectors of curvilinear functionals on the second order jet: sufficient efficiency conditions. Optim. Lett. (2011). doi:10.1007/s11590-011-0357-4

26. Sakai, T: Riemannian Geometry, vol. 149. Am. Math. Soc., Providence (1996)

27. Stampacchia, G: Formes bilineaires coercivities sur les ensembles coercivities sur les ensembles convexes. C. R. Math. Acad. Sci. Paris 258, 4413-4416 (1964)

28. Tang, G, Zhou, LW, Huang, NJ: The proximal point algorithm for pseudomonotone variational inequalities on Hadamard manifolds. Optim. Lett. (2012). doi:10.1007/s11590-012-0459-7

29. Udriste, C: Convex Functions and Optimization Methods on Riemannian Manifolds. Kluwer Academic, Dordrecht (1994)

30. Yao, Y, Noor, MA, Liou, YC: Strong convergence of a modified extra-gradient method to the minimum-norm solution of variational inequalities. Abstr. Appl. Anal. 2012, Article ID 817436 (2012)

31. Yao, Y, Noor, MA, Liou, YC, Kang, SM: Iterative algorithms for general multi-valued variational inequalities. Abstr. Appl. Anal. 2012, Article ID 768272 (2012)

doi:10.1186/1029-242X-2012-230

Cite this article as: Noor and Noor: Some algorithms for equilibrium problems on Hadamard manifolds. Journal of Inequalities and Applications 2012 2012:230. 